\title{
Effect of statistics of small numbers on the chemistry of trace species in atmospheric particles
}

\author{
Michael Mozurkewich \\ Chemistry Department and Centre for Atmospherıc Chemistry, York Unıversity, Toronto, Canada
}

\begin{abstract}
In models of reactions occurring in atmospheric aerosol particles, calculated concentrations of some trace species often imply fractions of a molecule per particle. When this occurs, averaging rate or equilibrium expressions over a large number of particles gives results that differ from bulk solution. Two different effects are demonstrated here. In the first, the confinement of ions in submicrometer drops suppresses the dissociation of weak acids and bases relative to bulk solution. For free radicals, there is an isolation effect that suppresses radical chain termination reactions when radical concentrations are low. Under extreme non-equilibrium conditions, half the drops contain one radical, even when bulk solution calculations indicate a concentration orders of magnitude smaller. This can greatly enhance the rates of radical chain reactions in drops relative to bulk solution. This latter effect has the potential to dramatically alter modelled rates of reactions in atmospheric particles.
\end{abstract}

\section{Introduction}

Atmospheric chemists now recognize the importance of reactions occurring in aqueous aerosol particles and are incorporating these reactions into computer models [Lelieveld and Crutzen, 1991; Chameides and Stelson, 1992; Fan and Jacob, 1992; Hanson et al., 1994, Lary et al , 1996; Sander and Crutzen, 1996]. In doing so, the aqueous phase chemistry is assumed to be the same as in bulk solution. However, calculated concentrations of some trace species imply fractions of a molecule per particle. For example, atmospheric fine particles have radii of the order of $0.1 \mu \mathrm{m}$. A single molecule in a droplet of this size is equivalent to a concentration of $4 \times 10^{-7} \mathrm{M}$. In any aqueous solution the concentration of either $\mathrm{H}^{+}$ or $\mathrm{OH}^{-}$will be less then this. Calculated concentrations of free radicals in atmospheric particles can be orders of magnitude smaller. Lelieveld and Crutzen [1991] calculate $\mathrm{OH}$ radical concentrations of $10^{-13}$ to $10^{-12} \mathrm{M}$ in $10 \mu \mathrm{m}$ radius cloud drops; this corresponds to 0.2 to 2 molecules per drop. Chameides and Stelson [1992] calculate $\mathrm{Cl}_{2}^{-}$and $\mathrm{Br}_{2}^{-}$concentrations of $10^{-11}$ to $10^{-9} \mathrm{M}$ in sea salt particles; for the $1.0 \mu \mathrm{m}$ particles of their low relative humidity case, this corresponds to 0.02 to 2 molecules per particle. Thus, for radicals in the submicrometer aerosol, there may be on average a sinall fraction of one molecule per particle.

The meaning of such a low average number of molecules per particle is that, at any given time, some particles will contain one molecule of the trace species and others will contain no molecules. Thus, the aqueous phase concentration of the trace species will vary considerably from one particle to another. If the trace species is produced or consumed in chemical reactions or if it can exchange with the gas phase, then there will be large variations in concentration within any one particle as a function of time. Such variatıons

\section{Copyright 1997 by the American Geophysica! Union.}

\section{Paper number 97GL03193.}

0094-8534/97/97GL-03193\$05.00 will not be directly observed in measurements since they will only apply when concentrations are much below the detection limits of available measurement techniques. However, reactive intermediates that play an important role in chemical transformations within particles may have very small average concentrations and so may be subject to these variations. In modelling the chemistry of atmospheric particles, these variations must be accounted for in averaging over the distribution of particles.

When aqueous phase concentrations vary widely from one particle to another, we must be careful about how we average over a large number of particles. Effects due to such variations have been previously discussed for systems subject to large fluctuations [Gıllespie, 1976; 1977] and for reactions occurring in micelles [Hatlee and Kozak, 1980; 1981a; 1981 b]. The consequences have been experimentally observed in micelle reactions [Moreno et al, 1996]. Here we consider the consequences for atmospheric particles.

Consider a bimolecular reaction occurring in small drops containing $n_{1}$ and $n_{2}$ molecules of the two reactants. The probability per unit time that a reaction occurs may be written as $k_{R} n_{1} n_{2} / V$ where $k_{\mathrm{R}}$ is the rate constant and $V$ is the volume of the drop. The probability averaged over a large number of drops is then $k_{R}<n_{1} n_{2}>\mid V$. However in applying bulk solution kinetics to this system, we write the probability as $k_{R}<n_{1}><n_{2}>/ V$.

This replacement of an average of a product with a product of averages is only valid if either there is no correlation between $n_{1}$ and $n_{2}$ or there is little variance in $n_{1}$ and $n_{2}$ over the distribution of particles. If $n_{1}$ and $n_{2}$ are large, the second condition applies as a consequence of the statistics of large numbers. This allows us to ignore correlations and fluctuations in treating kinetics and equilibria in bulk solutions. However, when $n_{1}$ and $n_{2}$ are small (of order unity or less), we can only use the product of averages if there is no correlation between the reactants. If two reactants have a common source (for example the ions produced by dissociation of a weak acid), they will tend to be positively correlated; then $\left\langle n_{1} n_{2}\right\rangle$ exceeds $\left.\left\langle n_{1}\right\rangle<n_{2}\right\rangle$ and the probability of reaction is increased.

In the following, we first consider the dissociation of weak acids since this case can be treated analytically and provides a basis for understanding more complex problems. We then give an example of how the statistics of small numbers may affect the chemistry of free radicals in particles. This latter case is potentially much more important. The full evaluation of its significance will require detailed examination of model results for instances where significant reactions involve two or more species for which the number of reactant molecules per particle is small.

\section{Acid-Base Equilibria}

To treat acid-base equilıbria, we assume that acid dissociation does not alter the number of acid molecules per drop. This condition is met if either the number of acid molecules are large compared to the number of ions formed or if the acid is maintained in equilibrium with a gas phase reservoir. The validity of latter condition, which will normally apply in the atmosphere, will be demonstrated later in this section. 
In general, we expect that atmospheric particles will contain a mixture of acids and bases; these will undergo a number of simultaneous acid-base equilibria producing a variety of anions. However, it is sufficient to simply consider the dissociation of a single acid. This is because the ratios of the average concentrations of the anions will be the same as in bulk solution. The reason is that each anion concentration, $\left[\mathrm{A}^{-}\right]$, is proportional to $[\mathrm{HA}] /\left[\mathrm{H}^{+}\right]$. Since the average concentration of acid, [HA], is assumed to be constant, it is independent of the acid-base equilibria. Thus, there is nothing to favour any one anion over the other anions and any change in chemistry in small drops relative to bulk solution must affect all anions in equal proportions. This statement does not apply to ions produced by strong electrolytes (e.g., $\mathrm{NaCl}$ ) since these are not directly involved in the acid-base equilibria. The fact that all anions are affected equally enables us to simplify the analysis by considering the dissociation of a single weak acid.'

We now consider an ensemble of water drops all of which have the same volume, ${ }^{2} V$. In any one drop there are an equal number of $\mathrm{H}^{+}$and $\mathrm{A}^{-}$ions but the number of pairs of ions varies from drop to drop. Let $p$ be the average number of pairs of ions expected per drop on the basis of bulk solution chemistry. Then

$$
p \equiv\left[H^{+}\right]_{\text {bulk }} V=\left[A^{-}\right]_{b u l k} V .
$$

At any given time, there will be a distribution of pairs of ions among the drops; let $N$, be the number of drops having $j$ pairs of ions. The number of pairs in a given drop will be constantly changing as a result of dissociation and recombination. Let $L_{j}$ be the probability per unit time that a recombination reaction will occur in a single drop containing $j$ pairs to form a drop with $j-1$ pairs. For the whole population, the rate of transition of drops with $j$ pairs to drops with $j-1$ pairs is $L_{1} N_{1}$. Let $F$ be the probability per unit time that dissociation will occur in a single drop and increase the number of pairs by one. The rate of transition of drops from $j-1$ to $j$ is $F N_{\text {j.1. }}$. At steady state these must balance, thus

$$
L, N_{1}=F N_{1-1} \text {. }
$$

If $k_{\mathrm{R}}$ is the rate constant of the reaction of $\mathrm{H}^{+}$with $\mathrm{A}^{-}$, we have

$$
L_{1}=k_{R}\left(\frac{j}{V}\right)^{2} V .
$$

$F$ must depend only on the volume of the drop and the probability of ionization per unit volume. The latter probability must be the same as in bulk solution since the ionization of an individual molecule should not depend on drop size. ${ }^{3}$ This point has been considered in detail by Gosele et al. [1979]. In bulk solution, the rate of ionization must balance the rate of recombination. Thus, using the definition of $p$ (equation 1)

$$
F=k_{R}\left[H^{+}\right]_{b u l k}\left[A^{-}\right]_{b u l k} V=k_{R}\left(\frac{p}{V}\right)^{2} V .
$$

Substituting equations (3) and (4) into equation (2), we obtain:

$$
N_{I}=\left(\frac{p}{j}\right)^{2} N_{t-1} \text {. }
$$

Repeated application of equation (5) provides the distribution function relative to $N_{0}$, the number of drops with no pairs of ions:

$$
N_{1}=\left(\frac{p^{21}}{j !^{2}}\right) N_{0} \text {. }
$$

The total number of drops, $N_{\mathrm{T}}$ is:

$$
N_{7}=\sum_{j=0}^{\infty} N_{j}=N_{0} \sum_{j=0}^{\infty} \frac{p^{2 \prime}}{j !^{2}} .
$$

The average value of $f^{n}$ is given by

$$
\left\langle j^{n}\right\rangle \equiv \frac{1}{N_{r}} \sum_{j=0}^{\infty} j^{n} N_{,}=\frac{1}{N_{T}} \sum_{i=0}^{\infty} \frac{j^{n} p^{2 l} N_{0}}{j !^{2}} .
$$

It is informative to evaluate $\left\langle j^{2}\right\rangle$ since this determines the average value of the product $\left[\mathrm{H}^{+}\right]\left[\mathrm{A}^{-}\right]$. Because the first non-zero term is that for $j=1$, we can rewrite equation (8) as

$$
\left\langle j^{2}\right\rangle=\frac{N_{0}}{N_{7}} p^{2} \sum_{i=1}^{\infty} \frac{p^{2(j-1)}}{(j-1) !^{2}} .
$$

Making a change of index, $i=j-1$, we have

$$
\left\langle j^{2}\right\rangle=\frac{N_{0}}{N_{T}} p^{2} \sum_{i=0}^{\infty} \frac{p^{2 i}}{l !^{2}}
$$

and, after using equation (7) to eliminate the summation,

$$
\left\langle j^{2}\right\rangle=p^{2}
$$

Since the average of $j^{2}$ is the same in a small drop as in bulk, one might be tempted to conclude that drop size has no effect. However, the rate of a first order reaction of one ion depends on $\langle p>$ rather than $\left\langle j^{2}>\right.$. The average of $J$ is given by

$$
\langle j\rangle=\frac{N_{0}}{N_{T}} \sum_{i=1}^{\infty} \frac{j p^{2 i}}{j !^{2}} .
$$

When $p$ is small enough that we need only consider terms up to order $p^{2}$ in the summations, this reduces to $\langle j\rangle \approx p^{2}=\left\langle j^{2}\right\rangle$. This is because if all drops have either $j=0$ or $j=1$, all powers of $j$ are equal to $J$. In this case, there is a large difference between the bulk result, $p$, and $\langle p\rangle$, the average over a collection of small drops. In the opposite limit of very large values of $p$, the summations are dominated by terms near the maximum of $N_{\mathrm{j}}$. For these terms, $j$ is approximately constant; thus $\langle p\rangle \approx j_{\text {mode }}$ the value of $j$ at the maximum. From equation (5), we see that $N_{\mathrm{J}}$ is a maximum when $j=p$; therefore, in the limit of large $j$, we obtain the bulk solution result, $<>\approx p$.

The transition between these limits is rather sharp. Numerical evaluation of the ratio $\langle p>/ p$ shows that it is within $2.5 \%$ of the bulk value of unity when $p \geq 10$ and is within $2 \%$ of the small drop limit, $p$, when $p \leq 0.2$. These numerical results also show that equation (12) is reasonably well approximated by the expression

$$
\langle\rangle\rangle \approx \frac{p^{2}}{\sqrt{1+p^{2}}} .
$$

Finite number of undissociated molecules. Now consider the case in which each drop contains a fixed small number, $n$, of acid molecules, HA. An example might be small clusters of water molecules condensed on a single acid molecule. In such a drop, the probability per unit time of forming an HA molecule by recombination of a pair of ions is still given by equation (3). The probabulity of forming a new pair of ions must be proportional to the number of undissociated HA molecules. Thus, this now depends on the number of pairs of ions, $j$. Equation (4) is therefore replaced by

$$
F_{1}=k_{R} K_{a} \frac{n-\jmath}{V} \text {. }
$$

where $K_{\mathrm{a}}$ is the acid dissociation constant and $k_{\mathrm{R}} K_{\mathrm{a}}$ is the first order rate constant for dissociation.

Using this in the above procedure we now obtain instead of equation (11),

$$
\frac{\left\langle j^{2}\right\rangle_{n}}{n-\langle j\rangle_{n}}=K_{a} V
$$

When $n$ and $J$ are large, we find \langle\rangle$\left.^{2}\right\rangle_{n}=p^{2}$ and \langle\rangle$_{n}=p$. Then equation (15) is identical to the usual equilibrium expression in bulk solution. However, this will not be true in general; for example, if $p$ is much less than 1 , then $\left\langle p>\approx\left\langle j{ }^{2}\right\rangle\right.$ as in the case where $n$ was not fixed. 
If HA can enter and leave the drop, the number of undissociated acid molecules, $n$, will be distributed among the drops according to a Poisson distribution. We may apply equation (15) to each $n$ in the distribution. Averaging over the distribution is straightforward; the result validates the assumption, made above, that equilibrium with a gas phase reservoir is equivalent to a very large number of undissociated molecules in the drop.

Application of results. We now have a procedure for correcting model calculations for the effect of drop size on the distribution of the various ions involved in acid-base equilibria. First, we carry out the acid-base calculations for bulk solution to obtain $\left[\mathrm{H}^{+}\right]$and, using equation (1), $p$. We then use either equation (12) or (13) to determine $\langle>>/ p$. This ratio is then applied to find the revised concentrations of $\left[\mathrm{H}^{+}\right]$and the various anions. If $p$ is large enough that the bulk result may be used, then the bulk result may also be used for all the individual anions; this includes anions with an average number per drop that is much less than unity. The reason for this is that the formation and loss of such anions has a negligible effect on $\left[\mathrm{H}^{+}\right]$since their concentration is much smaller than $\left[\mathrm{H}^{+}\right]$. Thus, their formation and loss is effectively first order and simple averages may be used. When the solution is basic, it is $\mathrm{OH}^{-}$rather than $\mathrm{H}^{+}$that should be regarded as the common ion and $\left[\mathrm{OH}^{-}\right]$should be used to compute $p$.

The effect of drop size on acid-base equilibria therefore depends only on the bulk solution $p H$ and the drop size. Figure 1 shows the drop size as a function of $p H$ over which the transition from bulk behaviour occurs. The effect of finite drop size increases as one moves below the lines shown; thus, the effect is most important for submicrometer particles with near neutral $p H$. Atmospheric particles with near neutral $p H$ are typically in the coarse mode with diameters greater than one micrometer [Prospero et al., 1983]; acid-base equilibria in these particles can be safely treated using a bulk solution approximation. Submicrometer particles usually have low $p H$ [Prospero et al , 1983], so the bulk approximation will usually be valid for these as well. Although there may be special cases for which the bulk treatment fails for atmospheric particles, this effect is more likely to be observed in laboratory experiments.

An example of this is the $\mathrm{HO}_{2}$ uptake experiments of Mozurkewich et al. [1987]. The observed uptake (their Figure 5) on partıcles with $p H=7$ was only slightly greater than on particles with $p H=5$.

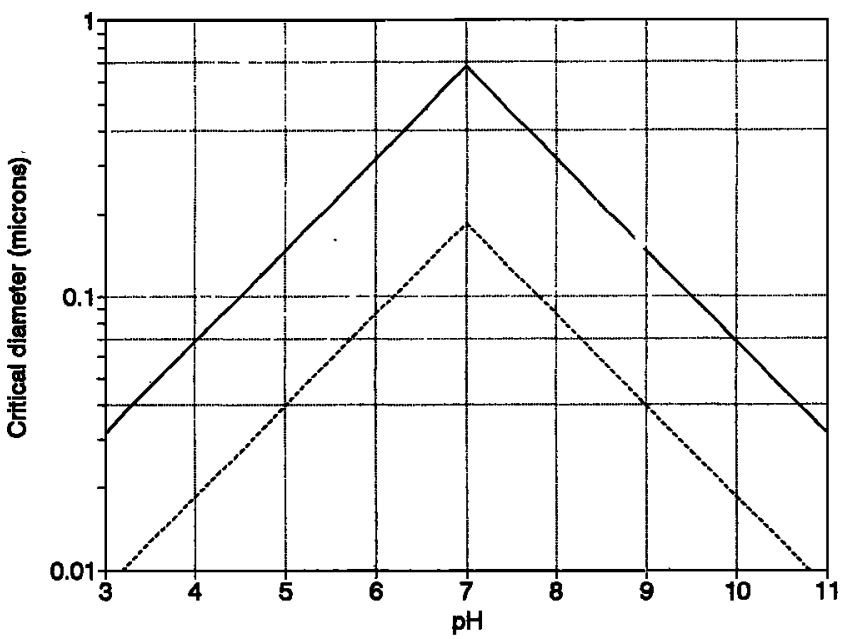

Figure 1. Drop diameters corresponding to different values of $p$, the average number of hydrogen or hydroxide ions per drop based on a bulk solution calculation. Curves are shown for $p=10$ (solid line) and $p=0.2$ (dashed line). Above the $p=10$ curve, the bulk solution result is valid; below the $p=0.2$ curve, it has broken down completely.
This was surprisıng since $\mathrm{HO}_{2}$ has a $p K_{\mathrm{a}}$ of 4.7 and should be a factor of 70 more soluble at the higher $p H$. This would dramatically increase its uptake. Since the particle radii were $0.055 \mu \mathrm{m}$, we have $p=4$ at $p H 5$ and $p=0.04$ at $p H 7$. The bulk calculation overestimates the dissociation of $\mathrm{HO}_{2}$ by a factor of 25 at $p H=7$. After taking this into account, the increase in solubility with the change in $\mathrm{pH}$ is only a factor of three. Thus the observed increase in uptake is much less than expected from a bulk calculation.

\section{Free Radical Reactions}

A significant effect in atmospheric particles is more likely to be encountered in the chemistry of free radicals. Radical-radical reactions provide a common sink of radicals and should produce a negative correlation in their concentrations. This will reduce the reaction rate. In particular, when radical concentrations are low, particles containing more than one radical are rare. If there are $n$ radicals in a particle, the rate of a radical-radical reaction is proportional to $n(n-1)$ since a molecule cannot react with itself. If $n$ is large, we may reasonably approximate $n(n-1)$ as $n^{2}$; this gives the rate law usually used for bulk solutions. But when $n$ is small, this approximation is seriously in error. Thus, radical concentrations and chain reactions in which radicals react with non-radical molecules may be much more efficient in small particles than in bulk solution.

Here we consider the case where a single radical is produced in a drop, for example by the transport of $\mathrm{OH}$ radicals into the drop from the gas phase. If the aqueous radicals are in equilibrium with the gas, the probability of finding two radicals in a drop is simply the square of the probability of finding one. However, the phases may not be in equilibrium. In solution the radical may react to form an ion that can not readily return to the gas phase. For example, in some recent experiments on $\mathrm{NaBr}(\mathrm{aq})$ particles (Anastasio and Mozurkewich, manuscript in preparation), any $\mathrm{OH}$ radicals entering the solution react according to

$$
\mathrm{OH}(\mathrm{g})+2 \mathrm{Br}^{-} \rightarrow \mathrm{OH}^{-}+\mathrm{Br}_{2}^{-} \text {. }
$$

This is the result of a rapid sequence of reactions [Mamou et al., 1977]; the rate determining step is collision of $\mathrm{OH}(\mathrm{g})$ with the particle surface. The thermodynamics of this reaction strongly favours $\mathrm{Br}_{2}^{-}\left(K_{\mathrm{cq}}=1.3 \times 10^{6} \mathrm{~atm}^{-1}\right.$ [Schwarz and Dodson, 1984]) and $\mathrm{Br}_{2}^{-}$rapidly disproportionates $\left(k_{2}=2 \times 10^{9} \mathrm{M}^{-1} \mathrm{~s}^{-1}\right.$ [Neta et al., 1988]),

$$
\mathrm{Br}_{2}^{-}+\mathrm{Br}_{2}^{-} \rightarrow \mathrm{Br}_{2}(\mathrm{~g})+2 \mathrm{Br}^{-}
$$

As a result, $\left[\mathrm{Br}_{2}(\mathrm{aq})\right]$ will tend to be much less than at equilibrium.

In the general case, radical loss occurs by both first order evaporation and second order reaction. The steady state equations (analogous to equation 2) for this system are simple to write out but difficult to solve analytically. However, numerical solutions to the kinetic equations can be readily obtained by the stochastic method of Gillespie [1976, 1977]. Averaging the kinetic results over time at long times provides the steady state solution.

Figure 2 shows the steady state number of $\mathrm{Br}_{2}^{-}$radicals per drop for both a bulk solution calculation, $n_{\mathrm{B}}$, and the stochastic calculation, $n_{\mathrm{S}}$. The bulk value, $n_{\mathrm{B}}$, is proportional to the square root of $\mathrm{OH}$ partial pressure, $P_{\mathrm{OH}}$. When $P_{\mathrm{OH}}$ is large enough that $n_{\mathrm{B}}$ is much greater than unity, the stochastic and bulk calculations give the same result; that is, $n_{\mathrm{S}}=n_{\mathrm{B}}$. But at small values of $P_{\mathrm{OH}}, n_{\mathrm{S}}$ levels off at a value of 0.5 , independent of $P_{\mathrm{OH}}$. This condition is maintained for several hours for a $50 \mathrm{~nm}$ drop. Eventually, $\mathrm{Br}$ is depleted, $\mathrm{OH}^{-}$ accumulates, and the equilibrium $\left[\mathrm{Br}_{2}{ }^{-}\right]$corresponding to a given $P_{\mathrm{OH}}$ (reaction $\mathrm{R} 1$ ) decreases. Only when the equilibrium value of $\left[\mathrm{Br}_{2}{ }^{-}\right]$begins to approach the steady state value does $n_{\mathrm{S}}$ begin to drop below 0.5 .

This somewhat unexpected result $\left(n_{S}=0.5\right)$ can be understood when we consider that under these conditions there is no evapora- 


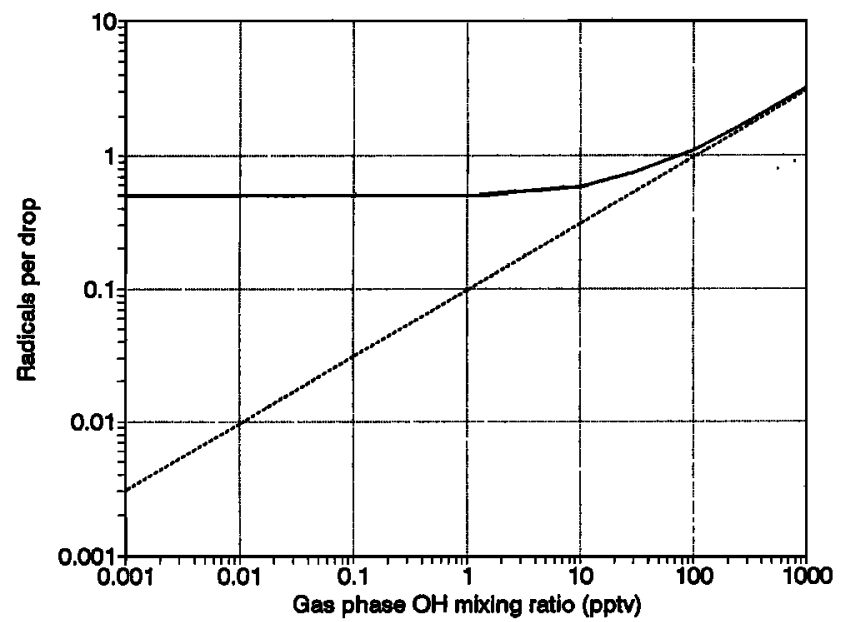

Figure 2. Average number of $\mathrm{Br}_{2}^{-}$radicals per drop at steady state. The drop radius is $50 \mathrm{~nm},\left[\mathrm{Br}^{-}\right]=5.4 \mathrm{M}$, total pressure is 1 atmosphere. The dotted line is computed using the bulk solution approximation and the solid line is from a stochastic computation that accounts for the discrete nature of the radicals.

tion of radicals from the drops. Thus, the only loss process for $\mathrm{Br}_{2}^{-}$ radicals is reaction ( $R 2$ ). In this case, we have aqueous radicals being both produced and destroyed at the mass transport limit. Any individual drop undergoes a simple cycle Starting with a drop containing no radicals, the arrival of an $\mathrm{OH}$ radical produces a drop with one $\mathrm{Br}_{2}$ - radical. The drop remains in this state until a second $\mathrm{OH}$ radical arrives. The resulting state with two $\mathrm{Br}_{2}{ }^{-}$radicals is very short lived; reaction (R2) quickly converts the drop back to the initial state with no radicals. At any instant in time, almost all drops have either zero or one radical and the average amount of time spent in each of these states is equal. Thus, the average number of radicals per drop, $n_{\mathrm{S}}$, is 0.5 . Increasing $P_{\mathrm{OH}}$ increases the rate at which a drop cycles through this sequence, but does not significantly alter $n_{\mathrm{S}}$.

Now consider the possibility that $\mathrm{Br}_{2}^{-}$participates in a chain reaction in which it is regenerated. An example might be the sequence [Song, 1996]

$$
\begin{gathered}
\mathrm{Br}_{2}^{-}+\mathrm{O}_{3}(\mathrm{aq}) \rightarrow \mathrm{O}_{3}^{-}+\mathrm{Br}_{2}(\mathrm{~g}) \\
\mathrm{O}_{3}^{-}+\mathrm{H}_{2} \mathrm{O}(\mathrm{l})-\mathrm{OH}(\mathrm{aq})+\mathrm{O}_{2}(\mathrm{~g})+\mathrm{OH}^{-} \\
\mathrm{OH}(\mathrm{aq})+2 \mathrm{Br}^{-} \rightarrow \mathrm{OH}^{-}+\mathrm{Br}_{2}^{-}
\end{gathered}
$$

This amounts to a radical catalyzed oxidation of $\mathrm{Br}$ by $\mathrm{O}_{3}$ At very low $P_{\mathrm{OH}}$, the average value of $\left[\mathrm{Br}_{2}{ }^{\circ}(\mathrm{aq})\right]$ can be orders of magnitude larger than expected from bulk solution. Then the rate of $\mathrm{Br}_{2}$ production by this chain reaction will be enhanced by the same factor.

There are a number of possible atmospheric implications of this potential for enhancement of radical chain reactions in particles. These include oxidant chemistry in cloud water [Lelieveld and Crutzen, 1991] sulphur oxidation in aerosol particles [Chameides and Stelson, 1992; Mozurkewich, 1995], and halide oxidation in aerosol particles [Sander and Crutzen, 1996; Mozurkewtch, 1995]. Since most radical concentrations are expected to be very small, these effects might matter even in coarse aerosol partıcles or in cloud drops. Model results should be closely examined for instances where significant reactions involve two or more species for which the number of reactant molecules per particle is small (of order unity or less). These reactions should then be treated using appropriate stochastic methods.

Acknowledgements. This work was supported by a grant from the Natural Science and Engineering Research Council of Canada 1 thank John Barker for brunging the work of Gillespie and of Moreno et al to my attention

\section{Notes}

1. This may be demonstrated rigorously by a straightforward expansion of the analysis given here.

2. For a distribution of sizes, this analysis can be applied separately to each range of sizes.

3. Here we ignore the relatively small changes due to the Kelvin effect.

\section{References}

Chameides, WL and A W Stelson, Aqueous-phase chemical processes in deliquescent sea-salt aerosols' A mechanssm that couples the atmospheric cycles of S and sea salt, J. Geophys Res , 97, 20,565-80, 1992.

Fan, S.-M. and D.J Jacob, Surface ozone depletion in Arctic spring sustaned by bromine reactions on aerosols, Nature, 359, 522-524, 1992

Gillespie, D T., A general method for numerically sımulatıng the stochastıc time evolution of coupled chemical reactions, J Comput Phys., 22, 403434, 1976.

Gıllespie, D.T , Exact stochastıc sımulation of coupled chemical reactions, $J$ Phys. Chem., 81, 2340-2361, 1977

Gosele, U, UKA Kleın and M Hauser, Diffusion-controlled reaction kınetics in micelles, Chem Phys Lett, 68, 291-295, 1979.

Hanson, D R., A.R. Ravishankara, and S Solomon, Heterogeneous reactions in sulfuric acid aerosols: A framework for model calculations, $J$ Geophys. Res, 99, 3615-29, 1994.

Hatlee, M D and J.J. Kozak, A stochastic approach to the theory of Intramıcellar kinetıcs I Master equation for irreversible reactions, $J$. Chem. Phys, 72, 4358-4367, 1980

Hatlee, M D. and J.J Kozak, A stochastic approach to the theory of intramicellar kinetics II Master equation for reversible reactions, $J$. Chem Phys., 74, 1098-1109, 1981a.

Hatlee, M D. and J J Kozak, A stochastic approach to the theory of intramicellar kınetics. III The homogeneous system limit, $J$ Chem Phys, 74, 5627-5635, $1981 \mathrm{~b}$.

Lary, D I, M P Chipperfield, R Toumi, and T Lenton, Heterogeneous atmospheric bromıne chemıstry, $J$ Geophys Res, I0I, 1489-1504, 1996

Lelıeveld, J. and PJ Crutzen, The role of clouds in tropospheric photochemistry, J. Atmos Chem, 12, 229-267, 1991.

Mamou, A, J Rabani, and D Behar, On the oxıdation of aqueous $\mathrm{Br}$ by $\mathrm{OH}$ radicals, studied by pulse radiolysis, $J$ Phys Chem., $81,1447-1448$, 1977

Moreno, M J , I.M G, Lourtie and E Melo, Stochastic effects on the timedependent rate constant of photodimerization of 12-(9anthroyloxy)stearic acid in micelles, $J$ Phys. Chem, 100, 18192-18200, 1996

Mozurkewich, M, PH McMurry, A Gupta, and J G. Calvert, Mass accommodation coefficient for $\mathrm{HO}_{2}$ radicals on aqueous particles, $J$ Geophys. Res, 92, 4163-4170, 1987

Mozurkewich, M., Mechanisms for the release of halogens from sea salt particles by free radical reactions, $J$ Geophys Res, 100, 14199-14207, 1995

Neta, P' R.E. Hure, and A.B Ross, Rate constants for reactions of inorganic radicals in aqueous solution, $J$ Phys. Chem Ref Data, 17, 1027-1284, 1988.

Prospero, J M, R J Charlson, V Mohnen, R. Jaenıcke, A. C Delany, J Moyers, $W$ Zoller, and $\mathrm{K}$ Rahn, The atmospheric aerosol system an overview, Rev Geophys. Space Phys., 2l, 1607-1629, 1983

Sander, R., and P.J Crutzen, Model study indicatıng halogen actıvation and ozone destruction in polluted arr masses transported to the sea, $J$ Geophys Res , 101, 9121-38, 1996

Schwarz, H A. and R W Dodson, Equilıbrsum between hydroxyl radıcals and thallium(II) and the oxidation potential of $\mathrm{OH}(\mathrm{aq}), J$. Phys Chem, $88,3643-3647,1984$

Song, R., Ph D Dissertatıon, Unıversity of Illınoss at Urbana-Champaign 1996

M Mozurkewich, Chemıstry Department and Centre for Atmospheric Chemistry, York University, 4700 Keele Street, North York, Ontario, M3J IP3, Canada. (nternet mozurkew@yorku ca)

(Received June 30, 1997; revised October 27, 1997, accepted October 31, 1997.) 